\title{
Diogenes syndrome in dementia: a case report
}

Luca Sacchi, Emanuela Rotondo, Sara Pozzoli, Alessio Fiorentini, Giuseppina Schinco, Clara Mandelli, Carlotta Coppola, Giorgio G. Fumagalli, Tiziana Carandini, Anna M. Pietroboni, Daniela Galimberti, Fabio Triulzi, Giorgio Marotta, Elio Scarpini, Matteo Cesari, Paolo Brambilla and Andrea Arighi

\section{Background}

Diogenes syndrome is a neurobehavioural syndrome characterised by domestic squalor, hoarding and lack of insight. It is an uncommon but high-mortality condition, often associated with dementia.

\section{Aims}

To describe the clinical features and treatment of Diogenes syndrome secondary to behavioural variant frontotemporal dementia (bvFTD).

\section{Method}

We describe a case of bvFTD in a 77-year-old man presenting with Diogenes syndrome.

\section{Results}

The patient's medical and psychiatric histories were unremarkable, but in recent years he had begun packing his flat with 'art pieces'. Mental state examination revealed confabulation and more structured delusions. Neuropsychological evaluation outlined an impairment in selective attention and letter verbal fluency, but no semantic impairment, in the context of an overall preserved mental functioning. Brain magnetic resonance imaging and positron emission tomography (PET) with fluorodeoxyglucose showed mild bilateral temporo-insular atrophy and hypometabolism in the left-superior temporal gyrus respectively. An amyloid PET scan and genetic analysis covering the dementia spectrum were normal. A diagnosis of bvFTD was made.

\section{Conclusions}

The clinical framing of behavioural symptoms of dementia such as hoarding poses a diagnostic challenge. This case illustrates the importance of a deeper understanding of Diogenes syndrome, leading to timelier diagnosis and effective therapeutic strategies.

\section{Keywords}

Diogenes syndrome; frontotemporal dementia; hoarding; collecting.

\section{Copyright and usage}

(C) The Author(s), 2021. Published by Cambridge University Press on behalf of the Royal College of Psychiatrists. This is an Open Access article, distributed under the terms of the Creative commons Attribution licence (http://creativecommons.org/ licenses/by/4.0/), which permits unrestricted re-use, distribution, and reproduction in any medium, provided the original work is properly cited.
Diogenes syndrome - named after the Greek philosopher and cynic is a neurobehavioural syndrome characterised by severe domestic squalor, pathological hoarding and lack of insight into the condition, ${ }^{1}$ the latter preventing the majority of patients from seeking medical help. First cases of elderly patients with self-neglect and extreme lack of hygiene were published by Dupré in $1925^{2}$ and Stevens in $1963^{3}$ and then more precisely described as a syndrome in 1966 by Macmillan \& Shaw, ${ }^{4}$ who called this condition 'senile breakdown'. Halliday et al in 2000 proposed domestic squalor, evidence of self-neglect, living alone, tendency to hoard and lack of concern for surroundings as the five defining features of the syndrome and they also developed the Environmental Cleanliness and Clutter Scale to assess squalor and hoarding. ${ }^{5}$ However, even in their study only $22 \%$ of individuals met all items on the scale and diagnostic criteria still lack consensus. ${ }^{5}$

The majority of cases occur in older adults (average age of 79 years) who live alone, although rare cases have been described in siblings and married couples. ${ }^{6}$ The current incidence of the full syndrome is not well-known: a retrospective observational French study found 1.6 cases per 10000 inhabitants; $25 \%$ of patients had the complete syndrome and $75 \%$ had the partial syndrome. Cipriani et al estimate an approximate annual incidence of $0.05 \%$ in people over the age of $60 .{ }^{8}$ In all probability, our society will be faced with more cases in the future owing to increased life expectancy and a consequent higher number of elderly individuals living alone.

Despite being an uncommon condition, diagnosis is paramount since Diogenes syndrome has been associated with increased morbidity and a $46 \% 5$-year mortality rate, ${ }^{9}$ with death commonly due to physical illnesses subsequent to self-neglect. Moreover, Diogenes syndrome poses ethical questions and legal challenges, such as finding a balance between autonomy and beneficence. ${ }^{10}$
Although the syndrome may occur as a single entity - so-called primary Diogenes syndrome - it is usually secondary to an underlying neuropsychiatric condition. Overall, Diogenes syndrome tends to be associated with psychosis, alcohol-induced disorder, affective disorder or obsessive-compulsive disorder (OCD) in younger individuals, whereas it is frequently associated with dementia in the elderly: clinical hoarding behaviour, often associated with selfneglect, and Diogenes syndrome are described in $23 \%{ }^{11}$ and $15 \%{ }^{12}$ respectively of older individuals with dementia. The unique combination of behavioural symptoms of behavioural variant frontotemporal dementia (bvFTD) may predispose to a high likelihood of developing Diogenes syndrome, which in fact occurs in up to $36 \%$ of people with bvFTD. ${ }^{13}$ We describe here a case of bvFTD presenting with Diogenes syndrome and subsequent follow-up of the patient.

\section{Ethics statement}

Informed written consent approved by the local Institutional Review Board was obtained from the patient, in accordance with specific national laws and the ethics standards laid down in the 1964 Declaration of Helsinki and its later amendments.

\section{Case report}

A 77-year-old dextral man was referred to the emergency department of our hospital by the police because he was found on the landing outside his flat, unkempt and dishevelled in personal appearance, after reportedly having lived there for 10 days. When questioned about this behaviour, he claimed that he was waiting for his nextdoor neighbour, who held a spare set of his flat keys, since he had accidentally locked his set in his garage. However, while he believed her to 


\begin{tabular}{|c|c|c|c|c|}
\hline Cognitive function and test & Reference value & Raw score & Corrected score & Interpretation \\
\hline \multicolumn{5}{|l|}{ Global cognitive functioning } \\
\hline Mini-Mental State Examination (MMSE) & $>23.8$ & $26 / 30$ & 28.03/30 & Normal \\
\hline \multicolumn{5}{|l|}{ Attention } \\
\hline Attentional Matrices & $>29.9$ & $31 / 60$ & $34.25 / 60$ & Inferior limit \\
\hline Trail Making Test (part A) & $<93$ & 50 & 19 & Normal \\
\hline \multicolumn{5}{|l|}{ Working memory } \\
\hline Letter verbal fluency & $>17.35$ & 4 & 13.4 & Impaired \\
\hline \multicolumn{5}{|l|}{ Language } \\
\hline Sartori Naming Test & $>50.36$ & $62 / 64$ & 37 & Normal \\
\hline semantic fluency & $>25$ & 27 & & Normal \\
\hline Semantic association (intracategorical) & $>17.92$ & $18 / 20$ & & Inferior limit \\
\hline \multicolumn{5}{|l|}{ Anterograde episodic memory } \\
\hline Short Story Task & $>4.75$ & $10 / 16$ & $10.75 / 16$ & Normal \\
\hline \multicolumn{5}{|l|}{ Visuospatial function } \\
\hline Clock Drawing Test & $>6.55$ & $13 / 13$ & $13 / 13$ & Normal \\
\hline \multicolumn{5}{|l|}{ Constructive apraxia } \\
\hline Rey Figure Copy & $>28.8$ & $30 / 36$ & $32.75 / 36$ & Normal \\
\hline
\end{tabular}

be on holiday, she had actually been living in a retirement home for 2 years. His medical history was significant only for a craniotomy, performed 16 years before, for the excision of a left parietal meningioma, with no residual neurological deficits, and hypertension, for which he did not take any drugs. He admittedly consumed more than a litre of wine per day but denied smoking. His psychiatric history was unremarkable, but in recent years he had begun packing his flat with 'art pieces'. An electrocardiogram performed in the emergency department revealed the presence of atrial fibrillation, and a chest radiography showed a right lower lobe consolidation, which were treated with oral medication because he refused any infusion therapy. He was initially admitted to the geriatric department and then moved to the psychiatric unit because of repeated instances of wandering and a quarrel with another homeless in-patient. When contacted, the patient's building manager reported behavioural changes over the past few years, and a recent history of pathological hoarding was confirmed by his general practitioner. A mental state examination revealed confabulation, along with a more structured delusion about being married to a counterterrorism spy, daughter of a 19th-century marshal, with whom he would communicate only non-verbally in order for her not to be discovered. Neuropsychological evaluation outlined an impairment in selective attention and letter verbal fluency retrieval, in the context of an overall preserved mental functioning, with a score of $26 / 30$ on the Mini-Mental State Examination (MMSE); a subsequent more thorough testing for language excluded semantic impairment (Table 1). Brain magnetic resonance imaging (MRI) showed a mild temporoinsular atrophy on both sides and calcifications in the left lenticular nucleus; fluorodeoxyglucose positron emission tomography (FDG$\mathrm{PET}$ ) revealed mild hypometabolism in the left superior temporal gyrus; amyloid PET did not reveal areas of increased radiotracer binding, ruling out an underlying amyloid brain deposition (Fig. 1). The search for expansion in the Chromosome 9 open reading frame 72 (C9orf72) gene gave negative results, and no mutations were detected through next generation sequencing (NGS) analysis. ${ }^{14}$ According to Rascovsky criteria, ${ }^{15}$ a diagnosis of bvFTD was made. In view of agitation and delusions, the patient was initially prescribed clotiapine, then shifted to zuclopenthixol and eventually to haloperidol, with only partial response. After the hospital admission, the patient was admitted to a care home.

\section{Discussion}

The exact pathogenesis and neuroanatomical basis of the full Diogenes syndrome are only hypothetical. In fact, no definite triggers to the condition have been identified and it is not clear whether solitude is a risk factor or a consequence of decision-making. Also, while hoarding is a major feature of Diogenes syndrome, there is no agreement on how to consider it in respect to other manifestations of the full syndrome (namely, squalor), to possibly related obsessive-compulsive behaviours and to other nosological entities such as OCD. The DSM- 5 considers hoarding disorder and OCD to be distinct clinically, neuroanatomically and therapeutically. Collecting in OCD is associated with anxiety and fear and often driven by obsessions or preoccupations, whereas hoarding disorder is characterised by distress on discarding possessions, leading to clutter. Results from voxel-based morphometry studies have detected significant structural differences both in people with OCD compared with controls ${ }^{16,17}$ and in people with hoarding disorder relative to those with OCD and to controls. ${ }^{18}$ Also, Hough et al found significantly greater dorsolateral prefrontal cortex activation during executive tasks in individuals with hoarding disorder compared with those with OCD. ${ }^{19}$

In contrast to hoarding disorder, acquired hoarding has been described both as a consequence of vascular lesions in the medial prefrontal or bilateral orbitofrontal $\operatorname{cortex}^{20}$ and in the context of dementia. ${ }^{21}$ Despite not being specific to any dementia subtype, hoarding appears to be much more common in bvFTD and semantic variant primary progressive aphasia (svPPA) - subtypes of FTD - occurring both in isolation and in the presence of additional obsessive-compulsive behaviours. ${ }^{22}$

Various explanations have been offered to account for the new onset of compulsive collecting in individuals with dementia.

Frontal lobe deficits are common in all the FTD subtypes and could clearly contribute to increased clutter due to a combination of disturbances. Finney et al reviewed five cases of Diogenes syndrome in individuals with bvFTD who exhibited hoarding accompanied by decline in self-care, other obsessive-compulsive behaviours and prominent executive dysfunctions. ${ }^{23}$ All these patients had bilateral or right predominant frontotemporal atrophy and hypometabolism, suggesting that compulsive collecting in these patients may be part of the environmental dependency syndrome in frontal disease, with specific involvement of a right frontolimbic-striatal system.

On the other hand, results from a quantitative MRI volumetric analysis of 47 individuals with FTD showed hoarding and obsessive-compulsive behaviours to be partially dissociable clinically and neuroanatomically, though highlighting an important role of the temporal lobes, particularly the left, in both disturbances. ${ }^{22}$ The study found that new or increased hoarding behaviour in people with FTD were more frequently associated with svPPA 

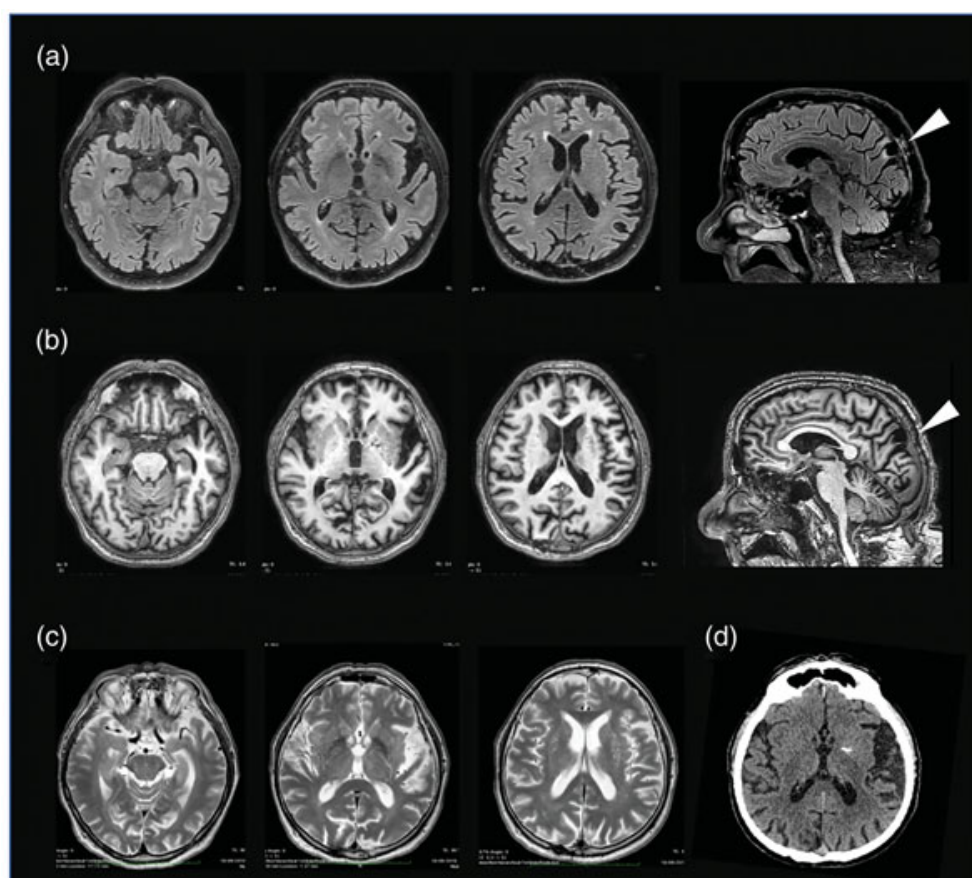

(d)

(e)

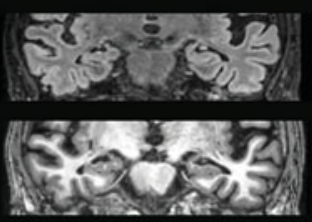

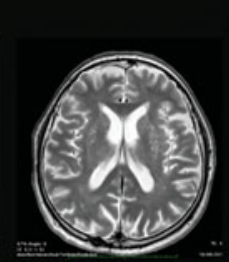

(f)

(g)

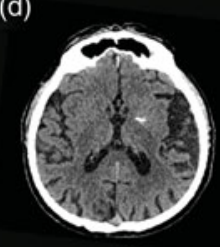

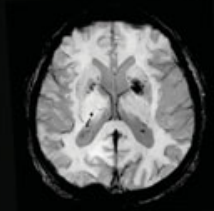

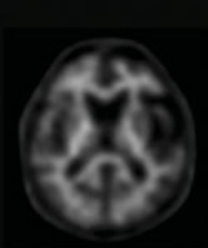

(h)

(a) Fluid attenuated inversion recovery (FLAIR) and (b) $\mathrm{T}_{1}$-weighted axial and sagittal (rightmost images) scans. (c) $\mathrm{T}_{2}$-weighted axial scans. (d) Computed tomography axial scan. (e) Coronal FLAIR section of temporal lobes (above) and coronal $T_{1}$ scans of temporal lobes (below). (f) Susceptibility weighted imaging (SWI) scan. (g) Amyloid positron emission tomography (PET) scan, negative for amyloid deposition. (h) fluorodeoxyglucose PET scans showing left superior temporal hypometabolism.

and predicted by left temporal atrophy. Left lateral temporal lobe grey matter loss also correlated with severity of obsessive-compulsive behaviours in a study conducted by Perry et al on 11 people with bvFTD. ${ }^{21}$ A plausible explanation for this finding is that deterioration of semantic organisation and storage secondary to degeneration of left temporal lobe structures, particularly in svPPA, may impair categorisation of objects, causing each to be considered unique and therefore non-discardable. Interestingly, in line with these newer data, our patient showed mild left superior temporal gyrus hypometabolism on the FDG-PET scan, much more limited in respect to the extensive frontotemporal hypometabolism in Finney et al's series ${ }^{23}$ and usually observed in bvFTD. ${ }^{24}$ However, to the best of our knowledge no previous studies specifically aimed at comparing patterns of cerebral glucose metabolism in individuals with FTD with and without hoarding are available to support our finding.

In conclusion, even if the development of the full Diogenes syndrome may require a more extensive and predominantly right-sided impairment of frontotemporal lobe connectivity and function, as seen in bvFTD, the occurrence of hoarding as an isolated finding in people with dementia, particularly in svPPA, along with newer literature suggests a pivotal role of left temporal lobe pathology in compulsive collecting, which our case seems to confirm.

Therapeutic management of Diogenes syndrome includes non-pharmacological and pharmacological approaches. Overall, both have proven difficult, since most patients deny their condition, avoid medical attention and exhibit poor therapeutic adherence.

Behavioural therapy-based skills seem beneficial. ${ }^{25}$ However, owing to the absence of randomised controlled trials, there are no guidelines on pharmacological interventions and no approved drugs, so that pharmacological interventions frequently depend on associated symptoms when present.

Many drugs have been tried in different studies, including selective serotonin reuptake inhibitors (SSRIs), first- and secondgeneration antipsychotics and anti-epileptics, with mixed results. Given the well acknowledged role of serotonin and dopamine in 
impulse control disorders and the approved use of SSRIs in the treatment of OCD, a therapeutic trial with molecules from one of these classes may be justified.

In fact, Finney et al reported some effect of high-dose sertraline in reducing collecting behaviours in two of their patients. ${ }^{23}$ Other authors found benefit on hoarding from other SSRIs ${ }^{26}$ and a combination of valproic acid and quetiapine in treating patients with bvFTD and secondary Diogenes syndrome. ${ }^{27}$ Lithium significantly improved one patient with long-standing Diogenes syndrome, although probably secondary to bipolar disorder, ${ }^{28}$ and risperidone reduced hoarding in an individual with Diogenes syndrome, but caused significant motor side-effects. ${ }^{29}$ Although not specifically tried for hoarding, trazodone has proven beneficial for behavioural symptoms in FTD. ${ }^{30}$

Our patient needed typical antipsychotics to manage agitation but quitting hoarding was mainly due to hospital admission and no specific treatment was tried. Admitting patients to hospital or moving them to another location is sometimes mandatory, but out-patient treatment through community care should be privileged if there is little risk to the patient or neighbours. Management should be conducted sensitively lest patients return to previous living conditions even more reluctant to receive medical aid.

\section{Implications}

This case, while illustrating the importance of suspecting Diogenes syndrome in elderly patients presenting with squalor and hoarding, especially in the context of dementia, supports recent evidence about the importance of the left temporal lobe in compulsive collecting pathogenesis. A deeper understanding of this condition, facilitating a timelier diagnosis, may lead to more effective pharmacological and non-pharmacological interventions, which in turn may reduce acute and chronic physical illness and improve social health outcomes.

\section{Luca Sacchi (DD), Dino Ferrari Center, University of Milan, Italy; Emanuela Rotondo (iD) Neurodegenerative Diseases Unit, Fondazione IRCCS Ca' Granda Ospedale Maggiore Policlinico, Milan, Italy; Sara Pozzoli, Department of Neurosciences and Mental Health, Fondazione IRCCS Cà Granda Ospedale Maggiore Policlinico, Milan, Italy; \\ Alessio Fiorentini, Department of Neurosciences and Mental Health, Fondazione IRCCS Cà Granda Ospedale Maggiore Policlinico, Milan, Italy; Giuseppina Schinco, Geriatric Unit, Fondazione IRCCS Ca' Granda Ospedale Maggiore Policlinico, Milan, Italy; Clara Mandelli, Geriatric Unit, Fondazione IRCCS Ca' Granda Ospedale Maggiore Policlinico, Milan, Italy; Carlotta Coppola, Geriatric Unit, Fondazione IRCCS Ca' Granda Ospedale Maggiore Policlinico, Milan, Italy; Giorgio G. Fumagalli $\mathbb{D}_{\text {, N Neurodegenerative }}$ Diseases Unit, Fondazione IRCCS Ca' Granda Ospedale Maggiore Policlinico, Milan, Italy; Tiziana Carandini (D), Neurodegenerative Diseases Unit, Fondazione IRCCS Ca' Granda Ospedale Maggiore Policlinico, Milan, Italy; Anna M. Pietroboni (D), Neurodegenerative Diseases Unit, Fondazione IRCCS Ca' Granda Ospedale Maggiore Policlinico, Milan, Italy; Daniela Galimberti (D), Neurodegenerative Diseases Unit, Fondazione IRCCS Ca' \\ Granda Ospedale Maggiore Policlinico, Milan, Italy; and Dino Ferrari Center, University of Milan, Italy; Fabio Triulzi, Neuroradiology Unit, Fondazione IRCCS Ca' Granda Ospedale Maggiore Policlinico, Milan, Italy; Giorgio Marotta, Nuclear Medicine Unit, Fondazione IRCCS Ca' Granda Ospedale Maggiore Policlinico, Milan, Italy; Elio Scarpini (D), Neurodegenerative Diseases Unit, Fondazione IRCCS Ca' Granda Ospedale Maggiore Policlinico, Milan, Italy; and Dino Ferrari Center, University of Milan, Italy; Matteo Cesari Geriatric Unit, IRCCS Istituti Clinici Scientifici Maugeri, University of Milan, Italy; Paolo Brambilla Department of Neurosciences and Mental Health, Fondazione IRCCS Cà Granda Ospedale Maggiore Policlinico, Milan, Italy; and Department of Pathophysiology and Transplantation, University of Milan, Italy; Andrea Arighi (1D) Neurodegenerative Diseases Unit, Fondazione IRCCS Ca' Granda Ospedale Maggiore} Policlinico, Milan, Italy

Correspondence: Andrea Arighi. Email: andrea.arighi@policlinico.mi.it

First received 3 Nov 2020, final revision 18 Dec 2020, accepted 23 Dec 2020

\section{Funding}

This research received no specific grant from any funding agency, commercial or not-for-profit sectors.

\section{Data availability}

The study data are available on reasonable request.

\section{Author contributions}

L.S. made substantial contributions to the acquisition of data and drafted the manuscript: ER. S.P., A.F., G.S., C.M., C.C., G.G.F., T.C., A.M.P., D.G.G., E.A.S., M.C. and P.B. made substantia contributions to the acquisition of data and revised the work; F.T. and G.M. made substantial contributions to the acquisition and interpretation of data and revised the work; A.A. made substantial contributions to the acquisition of data and drafted and revised the manuscript. All authors gave final approval of the version to be published and agreed to be accountable for all aspects of the work.

\section{Declaration of interest}

None.

\section{References}

1 Assal F. Diogenes syndrome. Front Neurol Neurosci 2018; 41, 90-7.

2 Dupré E. Les mendiants thésauriseurs. [The beggars hoarders.] In Pathologie de L'imagination L'émotivité. Payot, 1925: 429-44.

3 Stevens RS. Self-neglect in the elderly. Br J Geriatr Pract 1963; 2: 88-91.

4 Macmillan $D$, Shaw $P$. Senile breakdown in standards of personal and environmental cleanliness. BMJ 1966; 2: 1032-7.

5 Halliday G, Banerjee S, Philpot M, Macdonald A. Community study of people who live in squalor. Lancet 2000; 355: 882-6.

6 Halliday G, Snowdon J, Simpson B. Re: Diogenes syndrome in a pair of siblings. Can J Psychiatry 2005; 50: 567

7 Monfort J-C, Hugonot-Diener L, Devouche E, Wong C, Péan I. Le syndrome de Diogène et les situations apparentées d'auto-exclusion sociale: enquête descriptive. [Diogenes syndrome and related situations of social self-exclusion: descriptive survey.] Psychol Neuropsychiatr Vieil 2010; 8: 141-53.

8 Cipriani G, Lucetti C, Vedovello M, Nuti A. Diogenes syndrome in patients suffering from dementia. Dialogues Clin Neurosci 2012; 14: 455-60.

9 Clark ANG, Mankikar GD, Gray I. Diogenes syndrome. Lancet 1975; 305: 366-8.

10 Freckelton I. Hoarding disorder and the law. J Law Med 2012; 20: 225-49.

11 Hwang JP, Tsai SJ, Yang CH, Liu KM, Lirng JF. Hoarding behavior in dementia: a preliminary report. Am J Geriatr Psychiatry 1998; 6: 285-9.

12 Radebaugh TS, Hooper FJ, Gruenberg EM. The social breakdown syndrome in the elderly population living in the community: the Helping Study. $\mathrm{Br} J$ Psychiatry 1987; 151: 341-6.

13 Lebert F. Diogene syndrome, a clinical presentation of fronto-temporal dementia or not? Int J Geriatr Psychiatry 2005; 20: 1203-4.

14 Ghezzi L, Carandini T, Arighi A, Fenoglio C, Arcaro M, De Riz M, et al. Evidence of CNS $\beta$-amyloid deposition in Nasu-Hakola disease due to the TREM2 Q33X mutation. Neurology 2017; 89: 2503-5.

15 Rascovsky K, Hodges JR, Knopman D, Mendez MF, Kramer JH, Neuhaus J, et al. Sensitivity of revised diagnostic criteria for the behavioural variant of frontotemporal dementia. Brain 2011; 134: 2456-77.

16 Piras F, Piras F, Chiapponi C, Girardi P, Caltagirone C, Spalletta G. Widespread structural brain changes in OCD: a systematic review of voxel-based morphometry studies. Cortex 2015; 62: 89-108.

17 Subirà M, Cano M, de Wit SJ, Alonso P, Cardoner N, Hoexter MQ, et al. Structural covariance of neostriatal and limbic regions in patients with obsessivecompulsive disorder. J Psychiatry Neurosci 2016; 41: 115-23.

18 Yamada S, Nakao T, Ikari K, Kuwano M, Murayama K, Tomiyama $\mathrm{H}$, et al. A unique increase in prefrontal gray matter volume in hoarding disorder compared to obsessive-compulsive disorder. Plos One 2018; 13: e0200814.

19 Hough CM, Luks TL, Lai K, Vigil O, Guillory S, Nongpiur A, et al. Comparison of brain activation patterns during executive function tasks in hoarding disorder and non-hoarding OCD. Psychiatry Res Neuroimaging 2016; 255: 50-9.

20 Figee M, Wielaard I, Mazaheri A, Denys D. Neurosurgical targets for compulsivity: what can we learn from acquired brain lesions? Neurosci Biobehav Rev 2013; 37: 328-39.

21 Perry DC, Whitwell JL, Boeve BF, Pankratz VS, Knopman DS, Petersen RC, et al Voxel-based morphometry in patients with obsessive-compulsive behaviors in behavioral variant frontotemporal dementia: compulsions in FTD. Eur J Neurol 2012; 19: 911-7.

22 Mitchell E, Tavares TP, Palaniyappan L, Finger EC. Hoarding and obsessivecompulsive behaviours in frontotemporal dementia: clinical and neuroanatomic associations. Cortex 2019; 121: 443-53.

23 Finney CM, Mendez MF. Diogenes syndrome in frontotemporal dementia. Am J Alzheimers Dis Other Demen 2017: 32: 438-43. 
24 Cerami C, Dodich A, Lettieri G, lannaccone S, Magnani G, Marcone A, et al. Different FDG-PET metabolic patterns at single-subject level in the behavioral variant of fronto-temporal dementia. Cortex 2016; 83: 101-12.

25 Waserman JE, Hategan A, Bourgeois JA. Harnessing neuroplasticity in Diogenes syndrome: a proposed mechanism to explain clinical improvement. Gen Hosp Psychiatry 2014; 36: 761.e3-e5.

26 Saxena S, Brody AL, Maidment KM, Baxter LR. Paroxetine treatment of compulsive hoarding. J Psychiatr Res 2007; 41: 481-7.

27 Gálvez-Andres A, Blasco-Fontecilla H, González-Parra S, Molina JD, Padín JM, Rodriguez RH. Secondary bipolar disorder and Diogenes syndrome in frontotemporal dementia: behavioral improvement with quetiapine and sodium valproate. J Clin Psychopharmacol 2007; 27: 722-3.
28 Fond $\mathrm{G}$, Jollant $\mathrm{F}$, Abbar $\mathrm{M}$. The need to consider mood disorders, and especially chronic mania, in cases of Diogenes syndrome (squalor syndrome). Int Psychogeriatr 2011; 23: 505-7.

29 Herrán A, Vázquez-Barquero JL. Treatment of Diogenes syndrome with risperidone. Aging Neuropsychol Cogn 1999; 6: 96-8.

30 Lebert F, Stekke W, Hasenbroekx C, Pasquier F. Frontotemporal dementia: a randomised, controlled trial with trazodone. Dement Geriatr Cogn Disord 2004; 17: 355-9. 